Stefano Baia Curioni, Sara Bonini Baraldi Monica Calcagno, Costanza Cardullo, Donata Collodi

Francesco Crisci, Paola Dubini, Roberto Ferrari Francesco Giaccari, Daniele Goldoni, Alessandro Hinna Francesca Imperiale, Ludovica LeOne, Andrea Moretti Pamela Palmi, Luigi Maria Sicca Valentina TerlizZI, Luca ZaN

\title{
MANAGEMENT ARTI E CULTURE
}

Resoconto del primo anno del GSA Accademia Italiana Economia Aziendale

Luigi Maria Sicca e Luca Zan a cura di

Editoriale Scientifica

Napoli 
Tutti i diritti sono riservati

(C) 2014 Editoriale Scientifica srl

Via San Biagio dei Librai 39

80138 Napoli

www.editorialescientifica.com

info@editorialescientifica.com

ISBN 978-88-6342-675-5 


\section{Indice}

DIETRO LE QUINTE

Luigi Maria Sicca e Luca Zan

9 1. Introduzione

11 2. La preparazione del GSA

11 2.1. www.accademiaaidea.it, 16 marzo 2013

14 3. Gli incontri del primo anno di attività e metodo di discussione adoperato

15 3.1. L'opzione metodologica: la non neutralità dei mezzi rispetto allo scopo

17 3.2. Le otto ricerche proposte

19 4. Le prospettive per il secondo anno di vita del gruppo AIDEA ed impegni ad agire

20 5. Ringraziamenti

OSSERVAZIONE E PRATICHE DELLE ESPERIENZE ARTISTICHE:

QUESTIONE DI ETICHETTE ORGANIZZATIVE

Monica Calcagno, Costanza Cardullo e Luigi Maria Sicca

21 1. Obiettivo della ricerca

24 2. Contesto empirico studiato: danza verticale e Wanda Moretti

26 3. Metodologia utilizzata: osservazione partecipante

28 4. Perché quel contesto e cosa quel contesto può restituire agli studi aziendali

31 5. Perché quella metodologia e cosa quella metodologia può restituire agli studi aziendali

32 6. Conclusioni 
TRADURRE IN PRATICA UNA RIFORMA DEL FINANZIAMENTO REGIONALE ALLA CULTURA: ACCOUNTABILITY, PROCESSI DI CAMBIAMENTO ISTITUZIONALE E POLITICA CULTURALE Donata Collodi, Francesco Crisci e Andrea Moretti

37 1. Obiettivo della ricerca

38 2. Contesto empirico studiato

43 3. Metodologia utilizzata

45 4. Rilevanza e implicazioni di contenuto

46 5. Rilevanza e implicazioni metodologiche

48 6. Conclusioni

LA GESTIONE DELL'AZIENDA MUSEALE NELLA PROSPETTIVA DELLE LOGICHE ISTITUZIONALI Roberto Ferrari e Alessandro Hinna

51 1. Obiettivi della ricerca

52 2. Contesto empirico

55 3. Metodologia adottata

57 4. Rilevanza dei contenuti e implicazioni

59 5. Rilevanza metodologica e implicazioni

61 6. Conclusioni

CREATIVITÀ: ESTETIZZAZIONE DELL'ECONOMIA O LIBERAZIONE? Daniele Goldoni

63 1. Obiettivo della ricerca

63 2. Contesto empirico studiato

63 3. Metodologia utilizzata

66 4. Rilevanza e implicazioni di contenuto: perché quel contesto e cosa quel contesto può restituire agli studi aziendali

70 5. Rilevanza e implicazioni metodologiche: perché quella metodologia e cosa quella metodologia può restituire agli studi aziendali

72 6.Conclusioni 
COMMUNITY ENGAGEMENT NEI MUSEI PUBBLICI LOCALI

Francesco Giaccari, Francesca Imperiale e Valentina Terlizzi

75 1. Obiettivo della ricerca

76 2. Contesto empirico studiato

77 3. Metodologia utilizzata

80 4. Rilevanza e implicazioni di contenuto

84 5. Rilevanza e implicazioni metodologiche

85 6. Conclusioni

AlmoST FAMOUS: PENETRAZIONE COMMERCIALE E CONSACRAZIONE NEL SETTORE GLOBALE DELL'ARTE CONTEMPORANEA

Stefano Baia Curioni, Paola Dubini e Ludovica Leone

87 1. Obiettivo della ricerca

90 2. La base empirica

92 3. Metodologia utilizzata

93 4. Risultati degli studi

95 5. Perché quel contesto e cosa quel contesto può restituire agli studi aziendali

96 6. Perché quella metodologia e cosa quella metodologia può restituire agli studi aziendali

97 7. Conclusioni

UN MODELLO DI ANALISI ORGANIZZATIVA PER I DISTRETTI CREATIVI

Pamela Palmi

99 1. Obiettivo della ricerca

100 2. Contesto empirico studiato

101 3. Metodologia utilizzata

104 4. Perché quel contesto e cosa quel contesto può restituire agli studi aziendali

108 5. Perché quella metodologia e cosa quella metodologia può restituire agli studi aziendali

110 6. Conclusioni 
Progetto GIOCA MULTIMEDIALITÀ. ESPERIENZE NELL'UtILIZZO DI MATERIALI MULTIMEDIALI NELLA DIDATTICA E NELLA RICERCA Luca Zan e Sara Bonini Baraldi

113 1. Multimedialità: facile essere in ritardo?

114 2. Multimediale e didattica: un'esperienza diretta

117 3. Multimedialità e ricerca: l'esperienza GIOCA-Ricerche

125 Bibliografia

143 Indice dei nomi

145 Notizie sugli autori

147 Hanno scritto nella Collana punto org 